\title{
Descentralización y gestión ambiental en América Latina: un análisis de las publicaciones académicas*
}

Patricia Carrillo**

Antònia Casellas***

doi:I0.III44/Javeriana.cdri3-78.dgal

Recibido: 2016-06-16 Aprobado: 2016-08-29 Disponible en línea: 2016-12-20

Cómo citar este artículo: Carrillo, P. y Casellas, A. (2016). Descentralización y gestión ambiental en América Latina: Un análisis de las publicaciones académicas. Cuadernos de Desarrollo Rural, I3(78), 67-96. https://doi. org/10.III44/Javeriana.cdri5-78.dgal

* Artículo de investigación.

** Master en Estudios Interdisciplinarios en Sostenibilidad Ambiental, Económica y Social de la

Universidad Autónoma de Barcelona, España. Correo electrónico: patsiecarrillo@gmail.com. Orcid: http:// orcid.org/0000-000I-7010-5922

*** Profesora agregada adscrita a la Universidad Autónoma de Barcelona, España. Correo electrónico: antonia.casellas@uab.cat. Orcid: http://orcid.org/0000-0002-5252-1399 


\title{
Resumen:
}

La descentralización de la gestión ambiental implica la transferencia de poder y responsabilidades a nivel local. Aunque existen revisiones bibliográficas que versan sobre la descentralización y el medio ambiente, hay un vacío en el caso específico de Latinoamérica. Mediante la técnica del Systematic Quantitative Literature Review se observa que los patrones de la literatura revelan desequilibrios territoriales y temáticos en el análisis. Dichos resultados corresponden a la mayoría de estudios que se han centrado en Bolivia y Brasil; aproximaciones cuya concentración de investigaciones en la descentralización de la gestión forestal; dominio de los académicos del ámbito anglosajón, y valoraciones dispares en los resultados dependieron, a su vez, del país analizado y del enfoque disciplinar.

\section{Palabras clave:}

descentralización; gestión ambiental; gobernanza; revisión de literatura

\section{Decentralization and Environmental Management in Latin America: Analysis of Academic Journals}

\begin{abstract}
Decentralization of environmental management implies the transfer of power and responsibilities at the local level. Although there are bibliographic reviews dealing with decentralization and the environment, there is a gap in the specific case of Latin America. Through the Systematic Quantitative Literature Review technique, we observed that the literature patterns reveal territorial and thematic imbalances in the analysis. Said results correspond to a majority of studies that have focused on Bolivia and Brazil. These approaches, their focus on decentralization of forest management and their dissimilar result -which are within the domain of Anglo-Saxon academics- depended, in turn, on the country being reviewed and the disciplinary approach.
\end{abstract}

\section{Keywords:}

decentralization; environmental management; governance; literature review 


\section{Introducción}

La descentralización y, más específicamente, la descentralización de la gestión de los recursos naturales, es un proceso que se ha dado en la mayoría de los países en desarrollo y en transición desde mediados de 1980 (Larson y Soto, 2008). La difusión de la descentralización ha obedecido a dos razones. Por un lado, responde a una presión interna que en Latinoamérica se generó a partir del fin de los regímenes autoritarios $y$, por otro, es una estrategia promovida por organizaciones financieras internacionales y gobiernos donantes (Andersson y Van Laerhoven, 2007; Larson y Soto, 2008). La discusión y el análisis del proceso de descentralización que se vincula con la gestión de los recursos naturales es esencial, no solo por razones de conservación y de preservación, sino también porque la subsistencia y los ingresos de amplias capas de la población dependen de la gestión de los recursos naturales. A su vez, estos recursos son base de la riqueza que es fundamental para los gobiernos y las elites nacionales, por lo que históricamente la gestión ambiental ha sido un punto de conflicto (Larson y Ribot, 2004).

La literatura académica que versa sobre este tema es amplia, en cuyo sentido son notables múltiples revisiones bibliográficas alusivas a la gestión ambiental y a la descentralización (Larson y Ribot, 2004; Batterbury y Fernando, 2006; Lemos y Agrawal, 2006; Larson y Soto, 2008; Cohen y McCarthy, 2015). Sin embargo, no existe ninguna revisión de la literatura específica para el caso de Latinoamérica. Algunas de las revisiones mencionadas incluyen casos concretos de América Latina junto con ejemplos de África y Asia, lo que no permite estudiar en detalle la situación Latinoamericana. Asimismo, existen libros y artículos que versan sobre la descentralización en términos generales, pero no estudios o resultados que permitan hacer una valoración del tema en Latinoamérica (Rodríguez-Becerra y Espinoza, 2002; Gabaldón, 2009).

La literatura enfatiza en que la descentralización tiene resultados dispares dependiendo de los lugares en los que se desenvuelva, ya que diversos factores -la forma en la que se implementa, las relaciones con el gobierno central, las capacidades existentes a nivel local, la presión de organizaciones internacionales y donantes, las relaciones de poder a nivel local, entre otros factores- influyen en la manera como esta se desarrolla (Larson y Ribot, 2004). Teniendo en cuenta tal premisa, este artículo tiene como objetivo llenar un vació académico mediante la realización de una revisión sistemática de la literatura de la descentralización de la gestión ambiental en América Latina. Adicionalmente, cabe señalar que la mayor parte de la literatura publicada hasta la fecha se centra en el sector forestal, por lo que es necesario realizar una ampliación de temáticas e incluir en el análisis otros recursos naturales. La metodología 
utilizada para el estudio, el Systematic Quantitative Literature Review (SQLR) que es generado por Pickering y Byrne (20I4), y Rupprecht y Byrne (20I4), responde a las siguientes preguntas de investigación: ¿quién, dónde y cuándo se ha publicado sobre este tema?, ¿qué métodos se utilizan? y ¿qué temáticas se estudian?

La descentralización democrática, a la cual se hace aquí referencia, se diferencia de la descentralización administrativa o de la desconcentración en tanto que esta última se refiere a la transferencia de poder de las agencias gubernamentales centrales a las oficinas locales. De acuerdo con Ribot (2004) la descentralización democrática implica que las personas participan directamente en la toma de decisiones. En la literatura anglosajona se ha utilizado el término rescaling para referirse a este proceso de descentralización. De acuerdo con esta visión, scaling down involucra el paso de la toma de decisiones a instancias de gobernanza más locales, mientras que scaling out hace referencia al incremento de la participación de actores fuera del gobierno en la toma de decisiones ambientales (Cohen y McCarthy, 2015).

Rodríguez-Becerra y Espinoza (2002) apuntan que los procesos de descentralización que han tenido lugar en América Latina han correspondido a los marcos de dos modelos políticos: el del modelo federal -en Argentina, Brasil, México y Venezuela- y el del unitario -en los países restantes-. El análisis de los procesos de descentralización que se han dado en la gestión ambiental muestra que las diferencias existentes entre los países no se deben a estos modelos políticos, sino que corresponden a criterios internos de cada uno de ellos. Dentro del modelo federal, que rige el caso de Brasil, desde 1988 la gestión ambiental se ejerce en tres niveles de gobierno: federal, estadual y municipal; sin embargo, si bien en el caso de México los tres niveles participan en la gestión ambiental, el gobierno del país mantiene una mayor centralización en muchas áreas. De forma similar, mientras que en Venezuela se evidencia una desconcentración de direcciones a nivel estatal en el Ministerio del Medio Ambiente, Argentina cuenta con un organismo ambiental para cada provincia (Rodríguez-Becerra y Espinoza, 2002).

Por otro lado, los países unitarios cuentan, o bien con un ministerio como forma de organización ambiental que obedece a una desconcentración de competencias en oficinas a nivel regional, o con competencias parciales o totales descentralizadas a escala de gobiernos provinciales o departamentales. Así, mientras que Perú tiene un modelo de gestión pública altamente centralizado, Colombia ha implementado un proceso de descentralización en departamentos con la existencia de Corporaciones Autónomas Regionales como máxima autoridad ambiental a nivel regional. Un caso similar es el de Chile, que cuenta con una institucionalidad 
ambiental descentralizada territorialmente mediante las Comisiones Regionales de Medio Ambiente (Rodríguez-Becerra y Espinoza, 2002).

Si bien las características de la descentralización no se pueden clasificar a partir de modelos nacionales federales o unitarios, sí es posible identificar dos procesos diferentes que podríamos clasificar como top-down o bottom-up. Por una parte, la descentralización como proceso top-down es entendida como un procedimiento promovido por el gobierno para mejorar la gestión del sector público. Un ejemplo de ello se encuentra en Bolivia, en donde como consecuencia de la descentralización de la gestión forestal se han otorgado poderes restringidos a los gobiernos locales y una autonomía limitada a los usuarios locales, mientras que el gobierno central decide los derechos de propiedad (Larson y Soto, 2008). Por otro lado, si la descentralización se percibe no solamente como un proceso administrativo sino también como uno político, se la debe considerar como un proceso bottom-up en el cual se da la apertura de espacios de participación desde abajo: emersiones inducidas por movimientos sociales y gobiernos locales (Larson y Ferroukhi, 2003; Larson y Soto, 2008). Un ejemplo se puede evidenciar en Nicaragua, en donde a pesar de que las reformas hayan ocurrido en gran parte debido a la influencia de agencias internacionales, se ha gestado un proceso desde los gobiernos locales con colaboración de la sociedad civil (Batterbury y Fernando, 2006; Larson 2003b, 2004).

Con el fin de avanzar en la exploración desarrollada sobre los conocimientos existentes en torno a la descentralización y la gestión ambiental, el artículo se organiza a partir de cinco apartados. A esta introducción le sigue una sección en la que se abordan las características de la metodología SQLR. En seguida, se muestran los resultados que dan respuesta a las preguntas de investigación; resultados sintetizados mediante cuadros y gráficas. A partir de ese punto la discusión se desarrolla para finalmente concluir con un breve resumen.

\section{La SQLR como estrategia metodológica mixta}

El análisis bibliográfico propuesto utiliza la metodología SQLR de acuerdo con los preceptos de Pickering y Byrne (20I4). Este método incorpora una aproximación sistemática y cuantitativa, lo cual evita los sesgos que pueden darse cuando se utiliza una metodología narrativa en la que el investigador es quien evalúa la pertinencia de la literatura (Pickering y Byrne, 20r4). Asimismo, el método es pertinente, entre otras razones, porque permite revisar literatura tanto cualitativa como cuantitativa, lo que lo hace ideal para temas de medio ambiente caracterizados por su transdisciplinariedad. Asimismo, la SQLR se puede definir, 
por un lado, como un método cuantitativo que permite medir la cantidad de investigación realizada sobre un tema de acuerdo con el número de documentos encontrados. Sin embargo, a diferencia de los meta-análisis hasta tanto formulados, los resultados de la revisión de la literatura no corresponden a datos que permitan organizar un análisis estadístico (Rupprecht y Byrne, 20I4). En contraste, las indagaciones resultantes permiten documentar, entre otra información, la extensión geográfica de la literatura, los resultados obtenidos y los métodos utilizados. Este método permite valorar las diferentes combinaciones de ubicación, temas, variables, respuestas y, simultáneamente, identificar las brechas existentes en la literatura.

El SQLR se divide en un total de 15 pasos (figura I) los cuales se pueden clasificar en tres grandes bloques. El primero, la búsqueda sistemática de documentos, consiste en la formulación del tema, las preguntas de investigación y las palabras clave para las posteriores búsquedas en bases de datos y la depuración de la selección con base en la lectura y evaluación de los artículos (pasos i-5). El segundo bloque, que envuelve la creación de bases de datos, incluye su estructuración y la formulación de una prueba que envuelve una revisión que abarca el so \% de la literatura. Una vez que la base de datos se ha afinado, se introducen todos los documentos y se producen las tablas de resumen de la información obtenida (pasos 6-Io). En el último bloque (pasos II-I5) se procede a la redacción del artículo (Pickering y Byrne, 20r4).

\section{Búsqueda sistemática de documentos}

Paso 1. Definir el tema

Paso 2. Formular las preguntas de investigación

Paso 3. Identificar las palabras clave

Paso 4. Identificar las bases de datos y realizar la busqueda

Paso 5. Leer y evaluar las publicaciones

\section{Crear la base de datos}

Paso 6. Estructurar la base de datos

Paso 7. Introducir el $10 \%$ de los documentos

Paso 8. Probar y revisar las categorías

Paso 9. Introducir todos los documentos

Paso 10. Producir y revisar las tablas de resumen

\begin{tabular}{l} 
Redacción del artículo \\
\hline $\begin{array}{l}\text { Paso 11. Escribir la } \\
\text { metodología }\end{array}$ \\
Paso 12. Evaluar los \\
resultados clave y las \\
conclusiones \\
Paso 13. Escribir los \\
resultados y la discusión \\
Paso 14. Escribir la \\
introducción, el abtract y las \\
referencias. \\
Paso 15. Revisar el artículo \\
hasta que esté listo para ser \\
publicado
\end{tabular}

Figura 1. Pasos de la metodología SQLR

Fuente: elaboración propia a partir de Pickering y Byrne (20I4) 
En este artículo el método corresponde al análisis de la literatura que investiga el proceso de descentralización alusivo a la gestión del medio ambiente en América Latina. Por ello las preguntas de investigación hacen referencia a quién, dónde y cuándo se ha publicado, y exploran los métodos que se utilizan y qué temáticas se estudian.

Uno de los sesgos notables que se pueden observar en los artículos que utilizan el SQLR consiste en no realizar una revisión de la literatura en otros idiomas (Pickering y Byrne, 20I4). Siguiendo a los autores Rupprecht y Byrne (20I4), quienes utilizan varios idiomas para superar estas limitaciones, las búsquedas se realizan en inglés, español y portugués. Lo anterior permite ejecutar una búsqueda más completa y tener en cuenta los idiomas más pertinentes en la publicación latinoamericana.

Según Pickering y Byrne (20I4), en el tercer paso, que consiste en la identificación de las palabras clave, se recomienda hacer una selección que obedezca a las que fueron utilizadas en aquellos artículos frecuentemente citados; del mismo modo se recomienda el empleo de varios sinónimos con el fin de identificar la literatura relevante. Siguiendo estas recomendaciones, en esta investigación se realiza una búsqueda con palabras clave en tres idiomas y sus combinaciones, con el fin de crear las ecuaciones de búsqueda, incluyendo sinónimos para un mismo concepto (cuadro I).

Cuadro 1. Palabras clave en los tres idiomas de búsqueda

\begin{tabular}{lll}
\hline \multicolumn{1}{c}{ INGLÉs } & \multicolumn{1}{c}{ EsPAÑOL } & \multicolumn{1}{c}{ PorTUGUÉs } \\
\hline Decentralization & Descentralización & Descentralização \\
\hline Governance & Gobernanza & Governança \\
\hline Local / municipal government & Gobierno local / municipal & Governo local / municipal \\
\hline $\begin{array}{l}\text { Natural resource management } \\
\text { Environmental management }\end{array}$ & Gestión ambiental & Gestão ambiental \\
\hline $\begin{array}{l}\text { Natural resource policy/ } \\
\text { Environmental Policy }\end{array}$ & Política ambiental & Politica ambiental \\
\hline
\end{tabular}

FuENTE: elaboración propia

La búsqueda se realizó de manera sistemática en las bases de datos electrónicas Scopus, Web of Knowledge y Google Scholar. En las bases de datos electrónicas Scopus y Web of Knowledge se utilizaron solo las palabras clave en inglés, debido a que la búsqueda también muestra resultados en otros idiomas cuyos resumen y palabras claves están traducidos al inglés. En Google Scholar se realizaron búsquedas en los tres idiomas. Dado que el análisis se centra en Latinoamérica, se consideró 
necesario incluir también las bases de datos Scielo y Redalyc. Los términos de búsqueda utilizados en estas bases de datos fueron distintos, ya que los buscadores son más precisos en inglés cuando se trabaja con palabras clave que combinan varios términos. En el caso del portugués y el español, la búsqueda fue más efectiva a partir de la combinación de pocas palabras. Finalmente, cabe destacar que, tomando en consideración la diversidad de países latinoamericanos que han resultado envueltos en estas dinámicas, no se incluyeron como palabras claves los nombres de los países ni "Latinoamérica". En consecuencia, se procedió a seleccionar inicialmente un gran número de artículos que, en una segunda fase, fueron escrudiñados para identificar los artículos que se relacionan con Latinoamérica (cuadro 2).

Cuadro 2. Ecuaciones de búsqueda y número de registros encontrados

\begin{tabular}{|c|c|c|c|}
\hline ECUACIÓN DE BÚSQUEDA & $\begin{array}{l}\text { BASE DE } \\
\text { DATOS }\end{array}$ & $\begin{array}{l}\text { Número de } \\
\text { REgISTROS } \\
\text { SELECCIONADos }\end{array}$ & $\begin{array}{l}\text { Número de } \\
\text { REgistros } \\
\text { LAtinOAMÉrica }\end{array}$ \\
\hline $\begin{array}{l}\text { (decentralization) AND (governance } \\
\text { OR government) AND (forestry OR } \\
\text { natural resource OR environment) }\end{array}$ & Scopus & 44 & 27 \\
\hline $\begin{array}{l}\text { (decentralization) AND (governance } \\
\text { OR government) AND (forestry OR } \\
\text { natural resource OR environment) }\end{array}$ & $\begin{array}{l}\text { Web of } \\
\text { Knowledge }\end{array}$ & 26 & I6 \\
\hline $\begin{array}{l}\text { (decentralization) AND (governance } \\
\text { OR government) AND (forestry OR } \\
\text { natural resource OR environment) }\end{array}$ & Google Scholar & 94 & 17 \\
\hline $\begin{array}{l}\text { "Gestión ambiental”, "descentralización” } \\
\text { "Descentralização da gestão florestal” }\end{array}$ & $\begin{array}{l}\text { Redalyc, Scielo } \\
\text { y Google } \\
\text { Scholar }\end{array}$ & 38 & 38 \\
\hline
\end{tabular}

Fuente: elaboración propia

La búsqueda realizada correspondió al periodo de 2000 a junio de 2016, y permitió la obtención de referencias que equivalen a un total de 202 publicaciones descargadas en EndNote. Tras la inclusión de bibliografía adicional, que fue encontrada mediante la alusión a referencias y búsquedas específicas asociadas con los autores más citados, el total de documentos ascendió a 224. Los diferentes pasos de depuración de la base de datos permitieron la selección de 48 artículos académicos para su análisis (figura 2). 


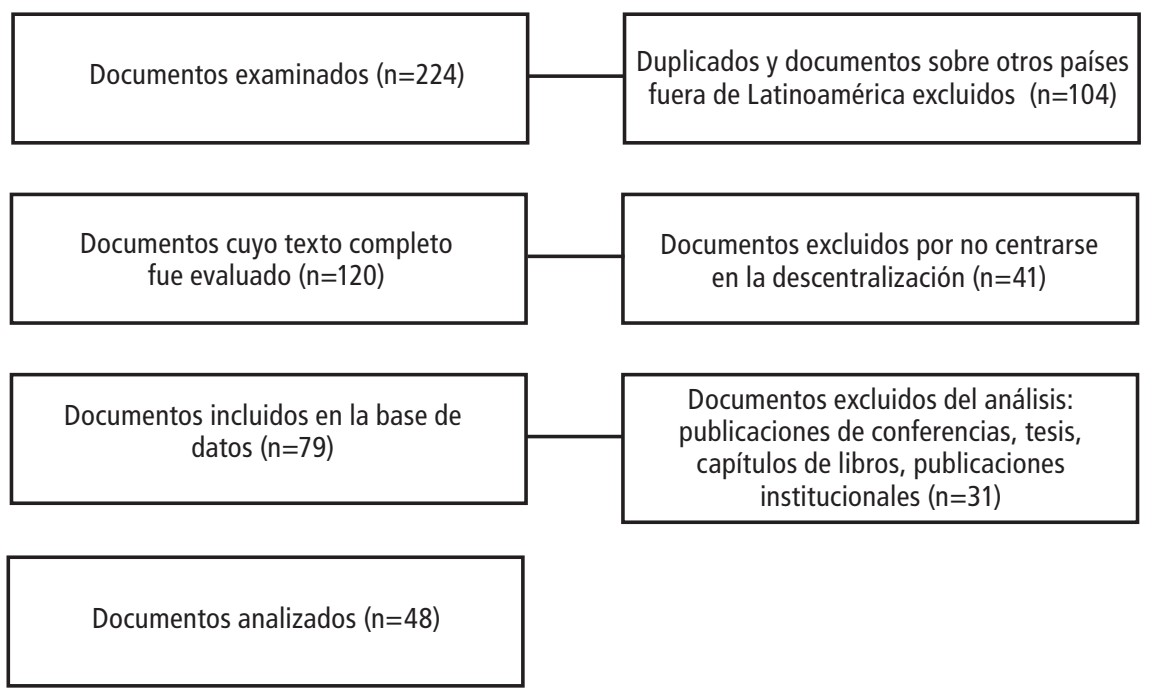

Figura 2. Número de documentos examinados e incluidos para el análisis Fuente: elaboración propia

En primer lugar, y como resultado de la exclusión de duplicaciones, los textos seleccionados finalmente correspondieron a un total de 48 artículos académicos. También se optó por excluir del estudio las tesis doctorales, libros, publicaciones institucionales y otras literaturas grises, con el objetivo de mantener la unidad de análisis que permiten los artículos académicos y la sistematización en la búsqueda de información; criterios fundamentales para el tipo de revisión bibliográfica que aquí se propone. Cabe enfatizar que resultados relevantes encontrados en la literatura se discuten en otras secciones de este artículo, incluso a pesar de no haber sido seleccionados para la revisión sistemática; en consecuencia, la mayoría de dichos resultados fueron incorporados en la introducción y en la contextualización del estudio.

Los 48 artículos seleccionados fueron clasificados sistemáticamente y además analizados según i) año de publicación, ii) país/área de estudio, iii) temática estudiada, iv) técnica metodológica utilizada, v) hipótesis o pregunta de investigación y vi) resultados principales. Asimismo se identificaron i) los autores y co-autores de los artículos, ii) el país de afiliación de los autores de acuerdo con la ubicación de la institución en la que se inscriben, iii) la tipología de revistas en las cuales se publican los artículos, iv) las disciplinas, y v) el país de publicación de la revista. Los resultados se presentan en cuadros y figuras siguiendo revisiones de la literatura recientes que utilizan la metodología SQLR (Guitart, Pickering y Byrne, 20I2; Rupprecht y Byrne, 20I4; Hirsch N'Guyen, Adrian-Kalchhauser y Burkhardt-Holm, 2016). 


\section{Resultados}

Con respecto a los patrones espaciales y lingüísticos, el análisis permite en primer lugar identificar la concentración de casos de estudio en unos pocos países. Los más estudiados, que corresponden a una diferencia significativa, son Bolivia con I9 artículos (39.6\%) y Brasil con I8 artículos (37.5\%). En segundo término aparecen Nicaragua, México y Guatemala, con 8 artículos cada uno (16.7 \% en cada caso). El restante 27.I \% se divide entre Perú, Honduras, Chile, Uruguay, Ecuador y Costa Rica (figura 3). Cabe destacar que el porcentaje no suma 100\%, ya que los artículos pueden estudiar más de un país. El análisis permite identificar lagunas espaciales significativas, como se observa en los casos de Colombia, Venezuela y Argentina.

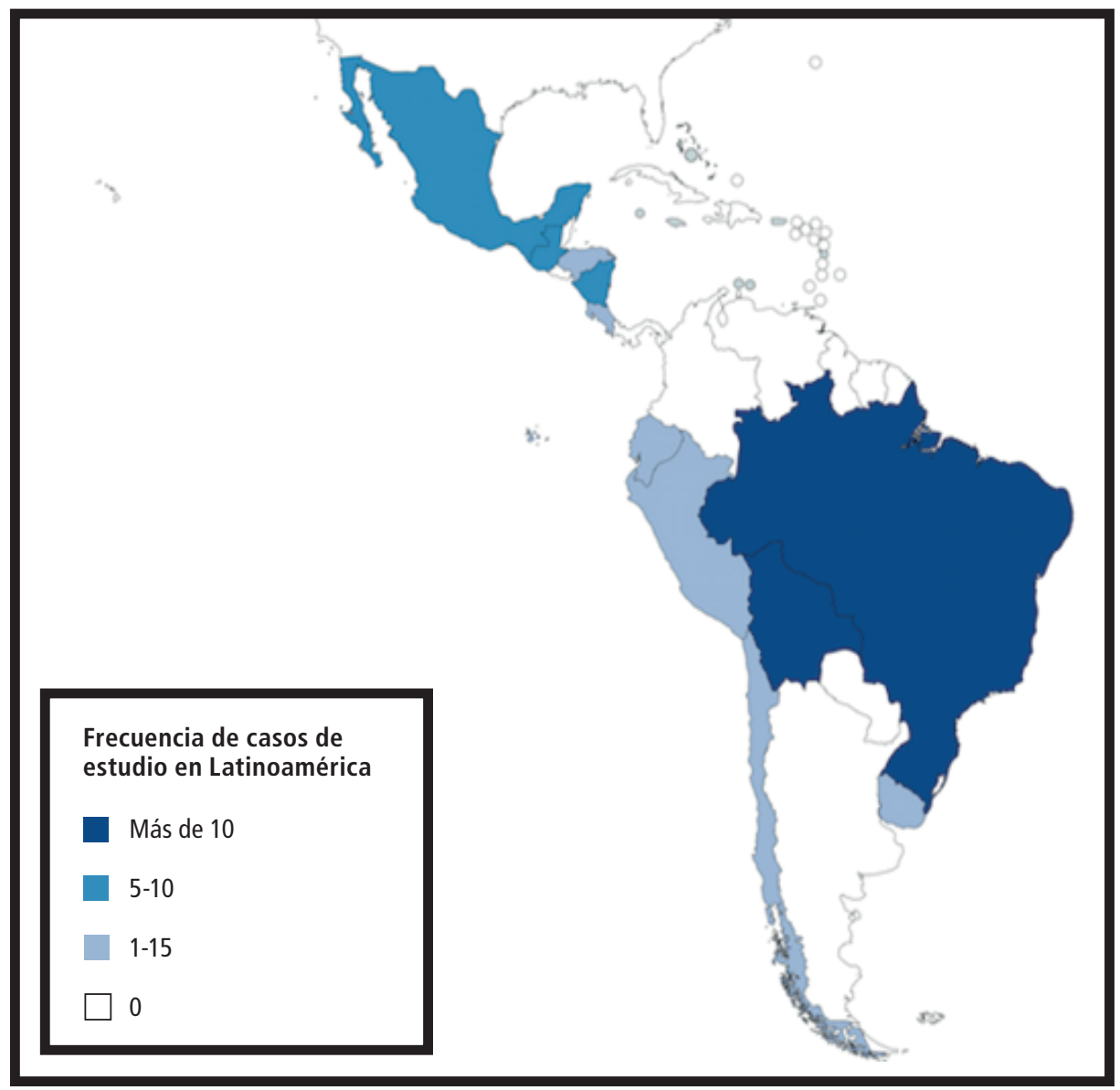

Figura 3. Mapa de América Latina con los países estudiados en la literatura en descentralización y medio ambiente

FUENTE: elaboración propia 


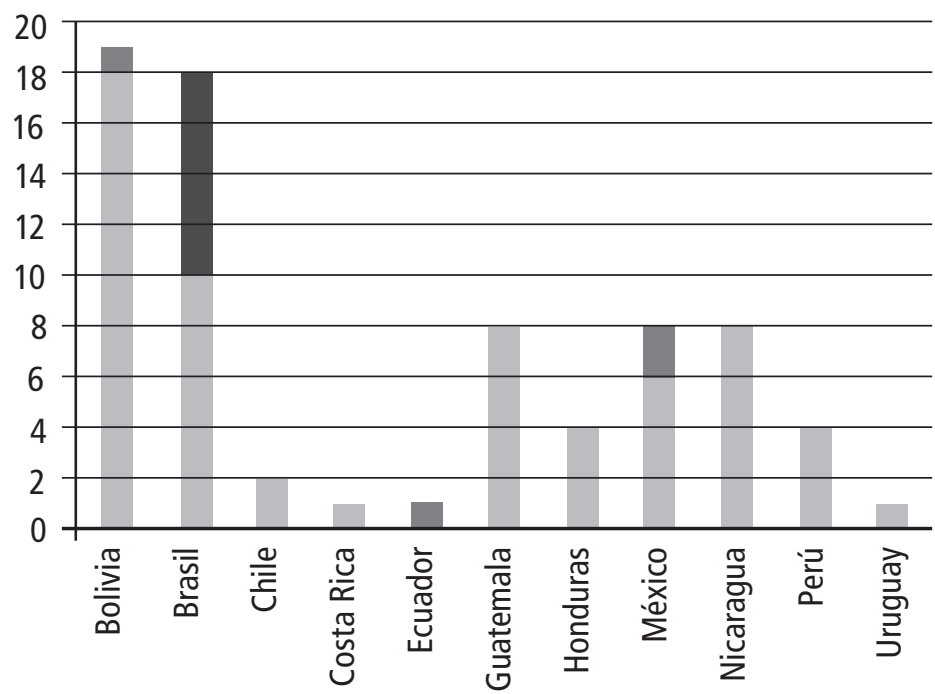

Español

Portugués

Inglés

Figura 4. Distribución geográfica y lingüística de los artículos en descentralización y medio ambiente

Fuente: elaboración propia

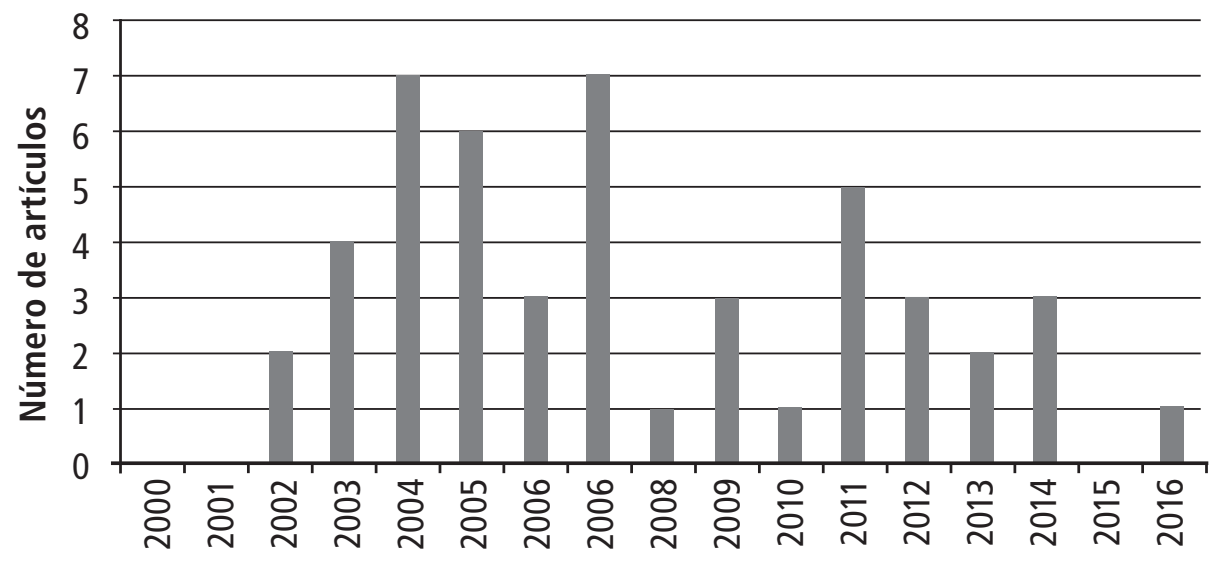

Año de publicación

Figura 5. Número de artículos por año de publicación

* Resultados hasta junio de 2016

Fuente: elaboración propia 
En segundo lugar, se observa que el inglés es el idioma dominante de las publicaciones ( $\mathrm{n}=36,75 \%)$, seguido a distancia por los artículos en portugués ( $\mathrm{n}=8$, I6.7 $\%)$ y, en un menor porcentaje, por el español (n=4, 8.3 \%). De acuerdo con lo observado en la figura 4, la búsqueda realizada en lengua portuguesa permitió acceder a más artículos centrados en el análisis de caso de Brasil. Asimismo, vale la pena remarcar que, en el caso de Ecuador, no se habrían obtenido tales resultados si la búsqueda no se hubiera realizado en español. Aquello ilustra el que el idioma de publicación sea relevante para hacer una revisión bibliográfica, y el que la presencia o la ausencia de ciertas áreas o países puedan estar vinculadas con razones lingüísticas.

Con respecto a las tendencias temporales de la literatura, y tal y como ya se ha indicado en la introducción, el proceso de descentralización se inicia en América Latina a mediados de 1980, y toma especial relevancia durante la década de 1990. Dado que se trata de un proceso largo, la reglamentación y puesta en marcha de la descentralización ha sufrido cambios y actualizaciones a lo largo del periodo de 2000 a 2016. Como se observa en la figura 5, los artículos académicos empezaron a surgir en el año 2002, con los picos de publicación correspondientes en los años 2004 y 2007 (n=7). Asimismo, tal como lo ilustra la figura aludida, las publicaciones han disminuido en los últimos años, con solo una concluida para el año de 2016 (resultados hasta junio 2016) y ninguna para el 2015.

El expuesto es un dato relevante porque, si bien el número de publicaciones ha disminuido notablemente en los últimos dos años para la región, en la actualidad se sigue publicando sobre otros países. Lo anterior abre preguntas importantes sobre las razones que pueden haber originado esta tendencia que, de mantenerse, debe ser analizada a mayor profundidad. Una de las explicaciones que se pueden proponer es que tal rasgo se vincule con la importancia del sector forestal, en el caso de Latinoamérica. En consecuencia, es plausible argumentar que las nuevas publicaciones que corresponden a la estrategia de REDD (Programa de Reducción de Emisiones de Carbono causadas por la Deforestación y la Degradación de los Bosques) capitalizan nuevos análisis. Así pues, vale la pena indicar que existen artículos que corresponden a la búsqueda, pero que no son incluidos en el análisis puesto que no cumplen los criterios de selección que se basan, originalmente, en la descentralización.

Con relación al país de afiliación de los autores principales y de los coautores - que se basa en la procedencia de la institución de afiliación del autor o autores, que es mencionada en la publicación-, se recalca el papel de liderazgo de los Estados Unidos $(\mathrm{n}=26,54.2 \%)$. Sin embargo, es importante destacar, en segundo lugar, la presencia de Brasil ( $\mathrm{n}=19,39.6 \%)$, y de México en tercera instancia (n=6, 12.5\%). Con la excepción de Brasil y México, los restantes trabajos que atañen a países latinoamericanos son 
minoritarios, y pertenecen a Bolivia, Nicaragua y Ecuador ( $\mathrm{n}=2,4.2 \%$ respectivamente), y Honduras y Guatemala (n=I, 2.I \%). Los países de afiliación Europea (n=8, I6.7 \%) incluyen a Francia, Reino Unido, Suiza, Finlandia, Dinamarca y Hungría. En el caso del último se realizó un artículo que hace comparaciones entre Hungría y México (Assetto, Hajba y Mumme, 2003). Es destacable que, a pesar de la proximidad idiomática e histórica, no se haya encontrado ninguna publicación realizada desde España. En el caso de los autores con afiliación asiática $(\mathrm{n}=2,4.2 \%)$, ambos resultados corresponden a un mismo artículo en el que se realiza una comparación entre Bolivia y Vietnam (Evans, De Jong, Cronkleton y Nghi, 20Io). [Para la lista completa de la afiliación de los autores y coautores ver Anexo A].

Un elemento destacable del presente estudio corresponde a la diversidad de publicaciones que se interesan en la temática de la descentralización, así como en la gestión ambiental que tiene lugar en América Latina. Los 48 artículos analizados se distribuyen entre 29 revistas. Las revistas que tienen tres o más publicaciones solo capturan el $35.4 \%$ del total de los artículos. Entre ellas se destacan World Development (n=II, $22.9 \%$ ), a la que le sigue European Journal of Development Research y Journal of Environment and Development (n=3, 6.3\%). Las revistas que publican dos artículos son cinco: i) Ambiente e Sociedade, ii) Gestión y Política Pública, iii) International Forestry Review, iv) Revista do Serviço Público, y v) Society and Natural Resources. Las 29 revistas están afiliadas a editoriales de los Estados Unidos (18.8 \%), Brasil (12.5 \%), Reino Unido (12.5 \%), Holanda (10.4 \%), México (4.2 \%) y Bolivia (2.1 \%). [Ver el Anexo A].

Finalmente, con relación al ámbito disciplinar, cabe subrayar que la mayor parte de los estudios que se enfocan en la descentralización y la gestión ambiental son publicados en revistas de Ciencias Sociales (47.9\%), seguidos por las Ciencias Ambientales (20.8 \%), Economía y Administración y Política Pública (ז0.4\%, respectivamente). El resto de publicaciones (27.I \%) se dividen entre las disciplinas de Geografía, Agricultura y Ciencias Biológicas, Planificación, Ingeniería, Artes y Humanidades, Psicología y Desarrollo. Los porcentajes no suman el $100 \%$, debido a que las revistas pueden abarcar más de una disciplina.

Para concluir este apartado, se puntualiza que, debido a los criterios de selección aplicados, los artículos que han sido objeto de análisis corresponden en su totalidad a casos empíricos. Todos implementan casos de estudio o discuten el sistema o parte del sistema de gestión ambiental de una o varias regiones o países. Las técnicas metodológicas incluyen, por orden de importancia: entrevistas (35.4\%), encuestas $(29.2 \%)$ y análisis estadísticos (29.2 \%). Cabe enfatizar que, tomados en su totalidad, los artículos que utilizan técnicas cualitativas son más numerosos que aquellos que se basan en metodologías cuantitativas o mixtas [ver cuadro 3]. 
Cuadro 3. Técnicas metodológicas utilizadas en artículos de descentralización y gestión ambiental

\begin{tabular}{lcc}
\hline Técnicas metodológicas & Número de artículos & Porcentaje De artículos (\%) \\
\hline Entrevistas & I7 & 35.4 \\
\hline Encuestas & I4 & 29.2 \\
\hline Análisis estadístico & I2 & 25.0 \\
\hline Observación & 5 & 10.4 \\
\hline Grupos focales & 5 & 10.4 \\
\hline SIG & 2 & 4.2 \\
\hline Método de escenarios & I & 2.1 \\
\hline Investigación acción participativa & I & 2.1 \\
\hline
\end{tabular}

FuENTE: elaboración propia

* El porcentaje no suma el ı००\% ya que los artículos contienen múltiples técnicas metodológicas.

\section{Discusión}

\section{Temáticas estudiadas}

Los artículos analizados abordan diferentes temáticas que pueden ser clasificadas en primera instancia de acuerdo con el recurso natural estudiado, por ejemplo: gestión forestal, gestión del agua y cuencas hidrográficas, etc. En primer lugar, cabe enfatizar que no todos los recursos naturales tienen una gestión descentralizada y, en el caso de los recursos naturales no renovables, como el petróleo o los minerales, la gestión permanece casi siempre bajo el control de la autoridad centralizada (Larson y Soto, 2008). En segundo lugar, se debe tener en cuenta que, tal y como lo apuntan Larson y Soto (2008, p. 2I4), en la descentralización de los recursos naturales "los intereses económicos y políticos específicos, las prioridades políticas y los problemas prácticos pueden variar en función del recurso natural en cuestión, en parte debido a las alternativas de derechos de propiedad asociados a las características del recurso" (Larson y Soto, 2008, p. 214).

Cabe remarcar que en el caso de América Latina, la temática forestal, como ya se ha indicado, domina el análisis, con un 52.I \% de los documentos, que corresponden a un total de 25 artículos. Como se dejó establecido en la metodología, la búsqueda incluyó palabras claves tales como resource managment, environmental o forestry, seleccionadas con el fin de obtener resultados de todos los recursos naturales. Específicamente, la palabra clave forestry se incluyó en los términos de búsqueda, ya que de acuerdo con las fuentes consultadas "los bosques son [...] el recurso que ha sido el más importante en los debates sobre políticas de descentralización de los recursos naturales y los más estudiados en la literatura de descentralización de los recursos naturales” (Larson y Soto, 2008, p. 214). Lo anterior queda corroborado en los resultados de esta búsqueda. 
$\mathrm{Al}$ enfocarnos en la descentralización de la gestión forestal, los países más estudiados son Bolivia y Guatemala -de acuerdo con leyes forestales implementadas desde la década de 1990-; Nicaragua -según la nueva ley forestal emitida en 2003-; Honduras -también de acuerdo con la ley forestal surgida en 2007- y Brasil -según la ley de concesiones forestales que apareció en 2006- (Larson, Pacheco, Toni y Vallejo, 2007b). En este ámbito, merecen atención especial los efectos de la deforestación vinculados con la descentralización; aspectos estudiado por autores de referencia, tales como Andersson y Gibson (2007), al igual que Paulson Priebe, Evans, Andersson y Castellanos (20I4).

La segunda temática que ha sido analizada en la literatura corresponde a la gestión descentralizada de cuencas hidrográficas (n=7, I4.6 \%). De estos estudios, seis abordan el caso de Brasil y uno el de México. El tercer bloque temático lo conforman los artículos que se dedican al medio ambiente como temática general (n=6, I2.5 $\%$ : textos que también estudian los ejemplos de Brasil y México y, adicionalmente, el de Perú. En el caso de Brasil, la investigación se centra en la discusión cuyo eje es el sistema nacional de medio ambiente que fue instaurado en el país, y sus correspondientes consejos municipales ambientales. Dos de estos artículos discuten a su vez las licencias ambientales que han correspondido a la gestión descentralizada (Agnes, Calegari, Gatto y Stangerlin, 2009; Nascimento y Bursztyn, 20II).

En cuarto lugar, vale la pena citar los estudios que se han centrado en territorios indígenas ( $\mathrm{n}=3,6.3 \%$ ), en concreto en Bolivia y Nicaragua. En el caso de Nicaragua, Larson y Lewis-Mendoza (20I2) analizan la gobernanza multinivel, lo que les permite concluir que las comunidades indígenas no pueden aprovecharla, ya que los actores más poderosos son los que dominan el proceso. En el ejemplo de Bolivia, Perreault (2005) examina los procesos de movilización que múltiples grupos de campesinos irrigadores desarrollan para contrarrestar las reformas del Estado. Por su parte, Uberhuaga, Larsen y Treue (20II) estudian la relación que existe entre la gestión forestal descentralizada y el nivel de vida de la población indígena de las tierras bajas.

Las temáticas restantes son tratadas por uno o dos artículos: por temáticas los textos corresponden a licencias ambientales, servicios públicos en el sector agricultura y recursos naturales, áreas protegidas, municipios con relación a la minería, y agua como una categoría más general, cada uno con n:2, 4.2\%. Por otro lado se encuentran cambios ambientales y el desarrollo rural, cada uno con n:I, 2.I \%. Dos de los artículos analizan municipios y recursos no renovables. El primero de ellos discute los consejos municipales ambientales en la zona de Medio Piracicaba, en Brasil, en donde el dinamismo demográfico y económico se asocia con la minería (Ferreira y Fonseca, 
20I4). El segundo estudia las instituciones de gobernanza ambientales en el Amazonas peruano, en donde se realizan exploraciones petroleras (Larsen, 20II).

Las áreas protegidas son estudiadas en dos artículos (n=2, 4.2 \%). En un primer estudio se analiza de qué modo varios organismos descentralizados implementan en Brasil políticas ambientales de manera eficaz (De Oliveira, 2002). En un segundo análisis se estudian las características de la participación de diversos actores en espacios de gobernanza ambiental en áreas protegidas de Carchi, Ecuador (Poats y Suárez, 2007). Por último, a nivel temático, en el caso del desarrollo rural se estudian los consejos municipales de desarrollo rural en México (Ticona, Vázquez, Cal y Mayor, y Meza, 2005). De este análisis, también cabe destacar que las temáticas de adaptación a cambios ambientales y desarrollo rural constan tan solo de un artículo que aborda la influencia de la descentralización en la adaptación al estrés ambiental en Uruguay (Thompson, 2016).

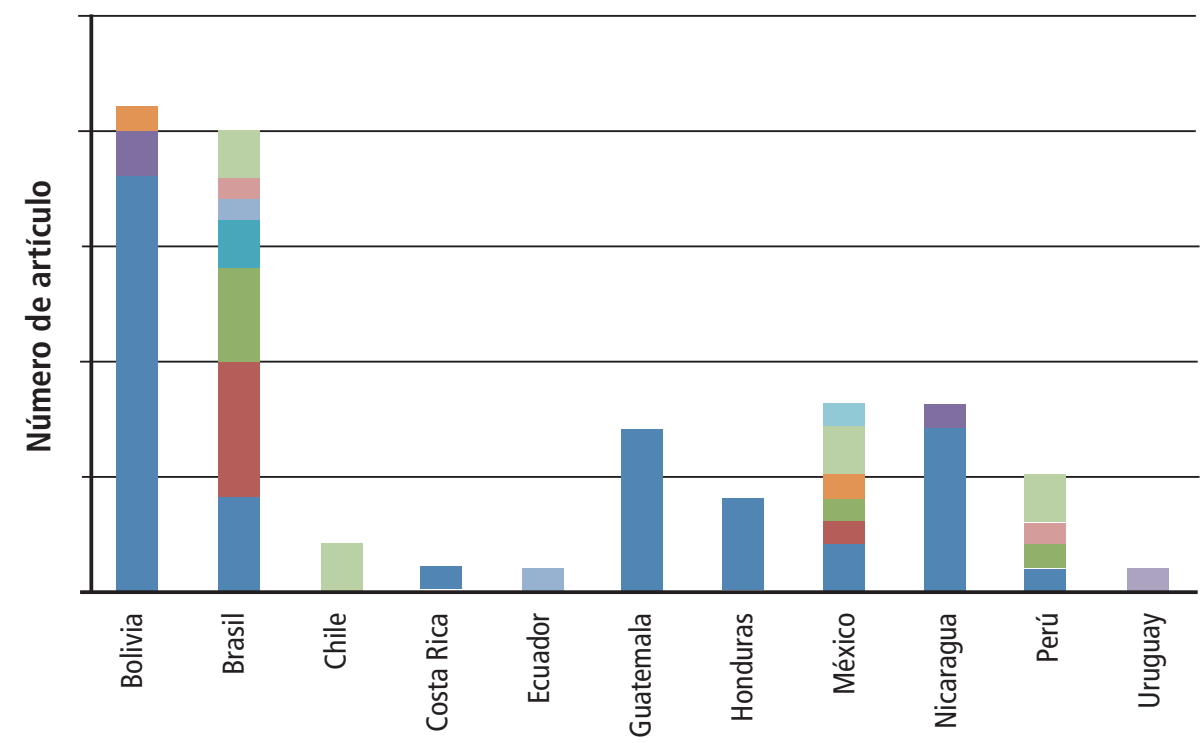

País de estudio

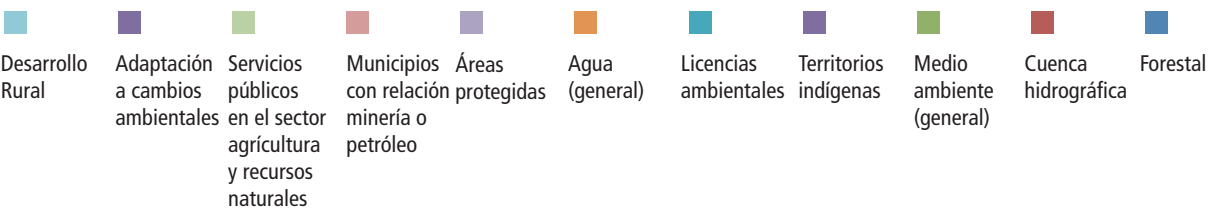

Figura 6. Temáticas estudiadas por país

* Cada artículo pueden contener una o más temáticas y uno o más países

Fuente: elaboración propia 
Como se puede observar en la figura 6, los países con mayor variedad de temáticas estudiadas son Brasil, México y Perú. Como se indicó en la introducción que versa sobre la descentralización en América Latina, los países se dividen entre aquellos que cuentan con un modelo federal y los que coinciden con uno unitario. De los países con modelo federal solo se encontraron artículos sobre Brasil y México. Los artículos que versan sobre Brasil presentan resultados diversos, pero denotan en tal caso un mayor poder sobre la gestión ambiental a nivel local que otros países.

\section{La descentralización a debate}

$\mathrm{Al}$ abordar el análisis de la literatura, otra opción que cabe considerar se organiza en función del grado de apoyo que se organiza en torno a los procesos de descentralización (Cohen y McCarthy, 2015). En esta sección, la discusión se estructura a partir de los artículos cuyos resultados aportan recomendaciones alusivas a cómo mejorar la gestión descentralizada y, a continuación, se detallan aquellos artículos que cuestionan la conveniencia de su aplicación.

Como ventajas de la descentralización, Rodríguez-Becerra y Espinoza (2002) destacan que esta i) acerca las decisiones para resolver los problemas ambientales correspondientes a los territorios en los cuales se generan - estados, provincias, municipios, etc.-; ii) crea oportunidades para incrementar la participación y la representación ciudadana; iii) da transparencia a las decisiones, y iv) aprovecha la competencia y la creatividad de instancias regionales, municipales y locales. En términos de la descentralización de la gestión ambiental, la literatura también pone de manifiesto múltiples desventajas. Entre estas se encuentra i) la falta de capacidad de los municipios para enfrentar ciertos problemas ambientales - capacidad tanto financiera como de recursos humanos-; ii) la falta de voluntad política, y iii) la captura de órganos de decisión por parte de las elites locales (Rodríguez-Becerra y Espinoza, 2002; Kauneckis y Andersson, 2009). Por su parte, Batterbury y Fernando (2006) advierten sobre las fallas que presentan en la práctica tales modelos normativos. De acuerdo con ello, la mayoría de intentos fracasan en la práctica por la captura de las elites o porque el gobierno central no cede suficiente poder.

Adicionalmente, Cohen y McCarthy (2015) destacan que las reformas de descentralización también se han llevado a cabo en nombre de las prácticas de buen gobierno, concepto ampliamente criticado como uno de los postulados de la reformas neoliberales.

En el análisis bibliográfico, dentro del primer grupo -artículos cuyos resultados son recomendaciones para mejorar la gestión descentralizada- se presta atención 
al estudio de las relaciones que se entablan entre los actores involucrados en la gobernanza ambiental y/o aquellos factores que inciden en los componentes de la escala local (ver Abers y Jorge, 2005; Abers y Keck, 20II; Andersson, 2003, 2004, 2013; Andersson, Gibson y Lehoucq, 2004, 2006; Andersson y Pacheco, 2005; Andersson y Gibson, 2007; Bartley, Andersson, Jagger, y Van Laerhoven, 2008; Gibson y Lehoucq, 2003; Kauneckis y Andersson, 2009; Larson 2002, 2003a; Larson, Pacheco, Toni, y Vallejo, 2007a, 2007b; Ravikumar, Andersson y Larson, 2013). El resumen de los actores identificados como relevantes, junto con sus interacciones, se encuentra esquematizado en la figura 7 .

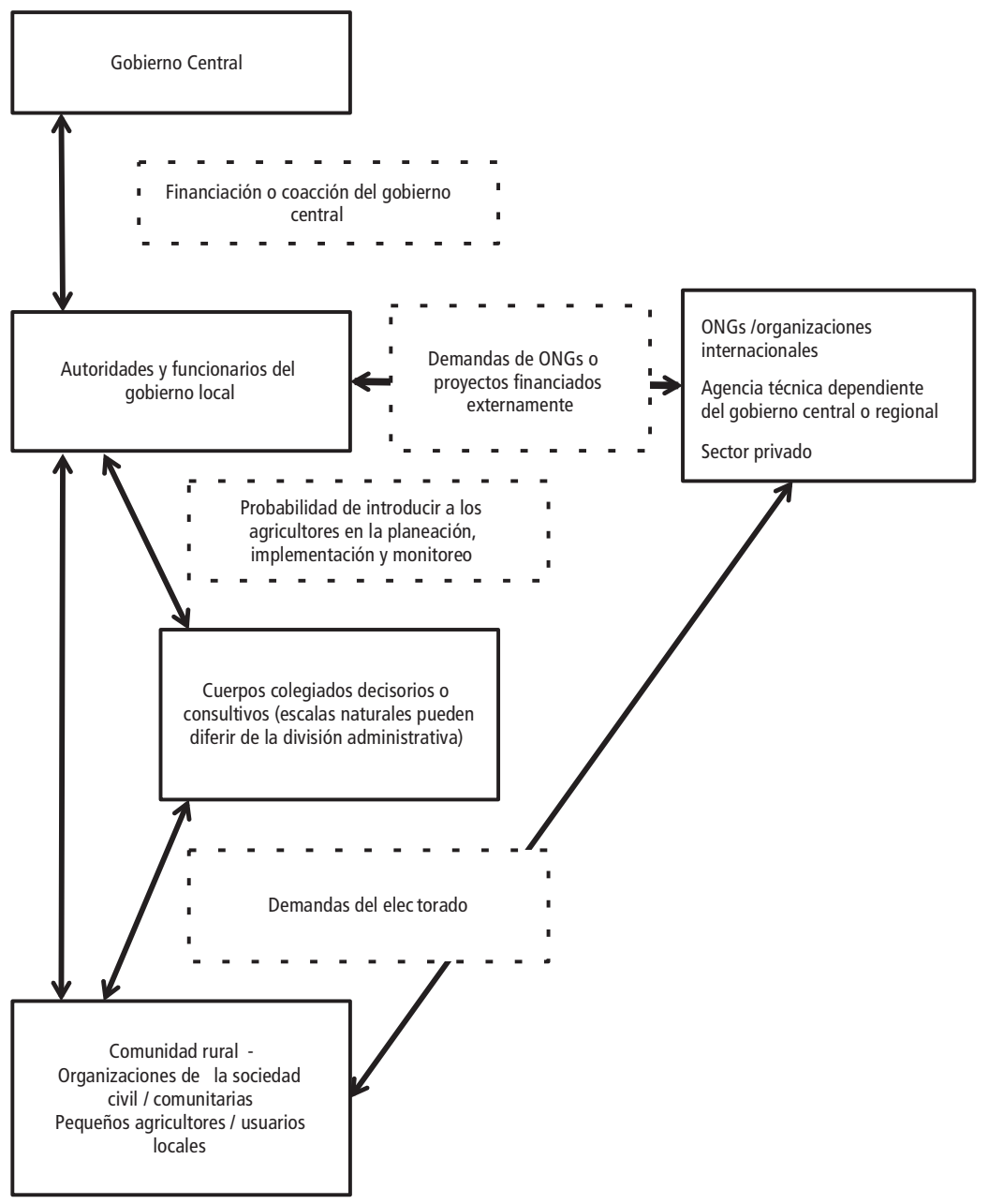

Figura 7. Esquema de actores e interacciones en la descentralización de la gestión ambiental

FuENTE: elaboración propia 
Estos artículos tienen alcances interesantes respecto a varios aspectos. En primer lugar, se abordan las características de los representantes locales y el grado de participación de la población en la toma de decisiones; los efectos o alteraciones que puede tener la descentralización en la pobreza, y la relación del gobierno local con el central o con otras instituciones -tales como ONGs, agencias dependientes del gobierno central, sector privado, que crean redes de confianza entre los stakeholders, además de reconocimiento social y político- para que la descentralización de las cuencas hidrográficas funcione. En la misma línea, Lemos y Farias de Oliveira (2004) incluyen la aparición de organizaciones bottom-up fuertes -independientes del Estado- como condición para lograr la gestión participativa. Una parte de la literatura apunta también a la necesidad de que los derechos de propiedad local sean establecidos o fortalecidos para mejorar las oportunidades de los pequeños productores (Larson, Pacheco, Toni y Vallejo, 2007a, 2007b; Coleman y Fleischman, 20I2). Como contrapartida, Coleman (20I4) apunta a que la vulnerabilidad de las familias ante las crisis es un factor a tener en cuenta en las políticas basadas en la participación. Asimismo, Evans, de Jong, Cronkleton y Nghi (2010) hacen hincapié en las limitaciones de la participación al señalar que las comunidades dependientes de los bosques suelen ser remotas y pequeñas, y que cuentan con deficiencias en la educación formal que condicionan sus habilidades en la negociación con otros stakeholders.

Respecto a la participación ciudadana, Andersson y Van Laerhoven (2007), y Kauneckis y Andersson (2009) concluyen que la presión y la organización de la sociedad civil son cruciales para fomentar la acción del gobierno local. Abers (2007) discute la importancia de construir una identidad colectiva -creando redes de confianza entre los stakeholders, además de reconocimiento social y político- para que la descentralización de las cuencas hidrográficas funcione. En la misma línea, Lemos y Farias de Oliveira (2004) incluyen la aparición de organizaciones bottom-up fuertes -independientes del Estado- como condición para lograr la gestión participativa. Una parte de la literatura apunta también a la necesidad de que los derechos de propiedad local sean establecidos o fortalecidos para mejorar las oportunidades de los pequeños productores (Larson, Pacheco, Toni y Vallejo, 2007a, 2007b; Coleman y Fleischman, 20I2).

Como contrapartida, Coleman (20I4) apunta a que la vulnerabilidad de las familias ante las crisis es un factor a tener en cuenta en las políticas basadas en la participación. Asimismo, Evans, de Jong, Cronkleton y Nghi (2010) hacen hincapié en las limitaciones de la participación al señalar que las comunidades dependientes de los bosques suelen ser remotas y pequeñas, y que cuentan con deficiencias en la educación formal que condicionan sus habilidades en la negociación con otros stakeholders. 
El segundo grupo de artículos analizados se centra en los resultados negativos de la descentralización, y pone en duda la conveniencia de su aplicación, del mismo modo que identifica claras limitaciones en ella. Ferreira y Fonseca (20I4), y Ticona et al. (2005) concluyen que en los consejos municipales ambientales que tuvieron lugar en Brasil, al igual que en los de desarrollo rural que se desenvolvieron en México, la participación se ha organizado en función de intereses individuales y de la cultura política, caracterizada a su vez por intercambios clientelares, corporativos y caciquiles, lo cual ha generado deficientes niveles de participación. En la misma línea, Wilder y Romero Lankao (2006) concluyen que la gestión descentralizada del agua y de los distritos de irrigación no ha demostrado ganancias en la eficiencia o beneficios ambientales para los usuarios, aunque en algunos contextos locales se han dado mejoras limitadas. Por su parte, Azevedo, Pasquis y Bursztyn (2007) enfatizan en que la poca capacidad de gobernanza de los subniveles estatal y local en Latinoamérica crean una descentralización del medio ambiente forzada de arriba que se dirige hacia abajo, y que corre el riesgo de parecer más una omisión del poder central que propiamente un logro democrático o una mejora en la gestión.

Otra limitación se identifica a través del análisis de la voluntad del poder central, que es investigada por Ribot, Agrawal y Larson (2006). En su estudio los autores utilizan un enfoque empírico comparativo, y muestran cómo gobiernos centrales de seis países - entre ellos Nicaragua y Bolivia- ponen en práctica una variedad de estrategias para obstruir la descentralización democrática de la gestión de recursos. De este análisis se desprende el que las dos estrategias principales que utilizan los gobiernos sean i) limitar el tipo de poderes que son transferidos y ii) elegir instituciones locales que sirvan y respondan a intereses centrales. Así, en el estudio de Nicaragua se demuestra que la prioridad del gobierno central es crear condiciones favorables para la industria y la inversión, mientras que la sociedad civil y los gobiernos locales son vistos como riesgos ya que pueden oponerse a los intereses de empresas extractivas. Otra investigación que se centra en Perú revela resultados similares, según los cuales la gobernanza municipal más que generar oportunidades para armonizar la gestión de los recursos, muestra claras contradicciones y limitaciones. En este caso las limitaciones se evidencian mediante los planes locales que se concentran en la industria el petróleo (Larsen, 20II). Tal y como ha sido analizado ampliamente, en el caso de las industrias extractivitas las decisiones importantes se toman fuera de los municipios (Bebbington en Larsen, 20II). En la misma línea crítica, los trabajos de Wittman y Geisler (2005) muestran que la descentralización de la gestión forestal que se desarrolla en Guatemala responde a una 
centralización difusa que genera un aumento del poder del Estado a nivel local, lo que pone en riesgo e incluso debilita las estructuras de gobernanza forestal exitosa de los pueblos y los medios de vida locales tradicionales.

Finalmente, otra crítica conecta la descentralización con las reformas neoliberales. El estudio de caso de la gobernanza del agua rural en Bolivia argumenta que la descentralización ha alterado la toma de decisiones, reestructurando la gobernanza ambiental (Perreault, 2005). Como aspecto complementario cabe mencionar que el resultado de estos estudios también pone en evidencia la aparición de una movilización regional y nacional de las asociaciones irrigadoras que se organizan para contrarrestar la descentralización de la gobernanza ambiental.

\section{Conclusión y dirección para futuras investigaciones}

La revisión sistemática de la literatura que se concentra en la descentralización y la gestión de los recursos naturales en América Latina muestra que la descentralización es un proceso complejo que tiene ventajas al igual que desventajas, y que en la práctica no funciona como es sugerido en la teoría. En líneas generales, la organización de la sociedad civil resulta crucial para demandar la acción del gobierno local, pero requiere de capacitaciones aptas para negociar con otros stakebolders. La descentralización democrática puede ser obstruida por los gobiernos centrales y tiende a ser vista como un riesgo que se opone a los intereses de las empresas extractivas. Asimismo, puede corresponder a una estrategia que permite aumentar el poder central a nivel local, y que debilita estructuras tradicionales de gobernanza exitosas. A su vez también puede ser interpretada como una manifestación de las reformas neoliberales que buscan conectar lo global, lo nacional y lo local, siguiendo las presiones e intereses de colectivos económicamente dominantes.

En primer lugar, se evidencian vacíos con relación a la distribución geográfica de la investigación. En ese sentido, los países más analizados son Brasil y Bolivia, mientras que se detecta una clara laguna de análisis en el caso de Argentina, Venezuela o Colombia. Por otra parte, los artículos que cubren información sobre países como Chile, Perú, Honduras, Uruguay, Ecuador y Costa Rica, son pocos.

En segundo lugar, y con respecto al origen de los investigadores, se observa una centralización de los estudios procedentes de los Estados Unidos, con una afiliación del $54.2 \%$ de los autores y coautores, lo que genera una narrativa dominante desde el ámbito anglosajón. Esto mismo se evidencia en el caso de los autores europeos, ya que 
la mayoría de las publicaciones (75\%) han sido realizadas en inglés. Aquello comporta el hecho de que el enfoque y la narrativa estén dominados por la cultura académica anglosajona. Por último, en cuanto al aspecto de autoría vale la pena destacar que en el presente estudio es evidente que el número de autores que han abordado el tema es reducido, lo que demuestra la falta de diversidad de las voces académicas.

En tercer lugar, cabe señalar que más del $50 \%$ de la literatura está concentrada en la temática forestal. Lo anterior demuestra la falta de profundización en otros recursos o bienes naturales. Por ejemplo, como destaca Larsen (20II), el análisis de las instituciones de gestión ambiental descentralizada no refleja las preocupaciones que conciernen a la explotación del petróleo. Asimismo cabe destacar que la presente investigación identifica una clara laguna con respecto al estudio de la descentralización y la gestión ambiental: brecha vinculada a los retos que genera el cambio climático en América Latina; un enfoque que sí se da en el estudio de otros países y regiones del mundo.

Finalmente, vale la pena enfatizar que la disminución del número de artículos publicados en los últimos años deja múltiples interrogantes abiertos. Entre estos, vale la pena establecer si la pérdida de interés de los académicos con respecto al tema se relaciona también con la de los políticos de la zona. Adicionalmente, aquello demuestra la evidente carencia de información de los resultados obtenidos por las reformas de descentralización a mediano y largo plazo, lo cual puede identificarse como un ámbito de posibles estudios futuros.

\section{Referencias}

Abers, R. (2007). Organizing for governance: building collaboration in Brazilian river basins. World Development, 35(8), I450-1463.

Abers, R. y Jorge, K. D. (2005). Descentralização da gestão da água: por que os comitês de bacia estão sendo criados. Ambiente e Sociedade, 8(2), 99-I24.

Abers, R. y Keck, M. (20II). Comitês de Bacia no Brasil: uma abordagem política no estudo da participação social. Revista Brasileira de Estudos Urbanos e Regionais, 6(I), 55-68.

Agnes, C. C., Calegari, L., Gatto, D. A. y Stangerlin, D. M. (2009). Uma discussão sobre a descentralização da gestão ambiental. Revista Científica Eletrônica de Engenharia Florestal, 8(I4), 53-73. 
Andersson, K. (2003). What motivates municipal governments? Uncovering the institutional incentives for municipal governance of forest resources in Bolivia. Journal of Environment and Development, I2(I), 5-27.

Andersson, K. (2004). Who Talks with Whom? The Role of Repeated Interactions in Decentralized Forest Governance. World Development, 32(2), 233-249.

Andersson, K. (2013). Local Governance of Forests and the Role of External Organizations: Some Ties Matter More Than Others. World Development, 43, 226-237. Andersson, K. y Gibson, C. C. (2007). Decentralized governance and environmental change: Local institutional moderation of deforestation in Bolivia. Journal of Policy Analysis and Management, 26(I), 99-123.

Andersson, K. y Pacheco, D. (2005). ¿Cómo hacer funcionar la gestión descentralizada de los recursos naturales? Gestion y Politica Publica, I4(I), 75-106.

Andersson, K. y Van Laerhoven, F. (2007). From local strongman to facilitator: Institutional incentives for participatory municipal governance in Latin America. Comparative Political Studies, 40(9), 1085-IIII.

Andersson, K., Gibson, C. C. y Lehoucq, F. (2004). The politics of decentralized natural resource governance. Ps-Political Science E- Politics, 37(3), 42I-426.

Andersson, K., Gibson, C. C. y Lehoucq, F. (2006). Municipal politics and forest governance: Comparative analysis of decentralization in Bolivia and Guatemala. World Development, 34(3), 576-595.

Assetto, V. J., Hajba, E. y Mumme, S. P. (2003). Democratization, decentralization, and local environmental policy capacity: Hungary and Mexico. The Social Science Journal, 40(2), 249-268.

Azevedo, A., Pasquis, R. y Bursztyn, M. (2007). A reforma do Estado, a emergência da descentralização e as políticas ambientais. Revista do Serviço Público, $58(\mathrm{I}), 37-55$.

Bartley, T., Andersson, K., Jagger, P. y Van Laerhoven, F. (2008). The contribution of institutional theories to explaining decentralization of natural resource governance. Society and Natural Resources, 2I(2), 160-174.

Batterbury, S. P. y Fernando, J. L. (2006). Rescaling governance and the impacts of political and environmental decentralization: An introduction. World Development, 34(II), I85I-1863.

Bebbington, A. (2006). La glocalización de la gobernanza ambiental: relaciones de escala en los movimientos socio ambientales y sus implicaciones para la gobernanza ambiental en zonas de influencia minera en el Perú y el Ecuador. Manchester: Universidad de Manchester, Escuela de Medio Amibiente y Desarrollo. 
Brannstrom, C. (2004). Decentralising water resource management in Brazil. European Journal of Development Research, I6(I), 214-234.

Cohen, A. y McCarthy, J. (2015). Reviewing rescaling: Strengthening the case for environmental considerations. Progress in Human Geography, 39(I), 3-25.

Coleman, E. A. (20I4). Behavioral Determinants of Citizen Involvement: Evidence from Natural Resource Decentralization Policy. Public Administration Review, 74(5), 642-654.

Coleman, E. A. y Fleischman, F. D. (20I2). Comparing Forest Decentralization and Local Institutional Change in Bolivia, Kenya, Mexico, and Uganda. World Development, 40(4), 836-849.

De Oliveira, J. A. (2002). Implementing environmental policies in developing countries through decentralization: The case of protected areas in Bahia, Brazil. World Development, 30(10), 1713-1736.

Evans, K., de Jong, W., Cronkleton, P. y Nghi, T. H. (2010). Participatory methods for planning the future in forest communities. Society and Natural Resources, 23(7), 604-619.

Ferreira, C. M. y Fonseca, A. (20I4). Análise da participação popular nos Conselhos Municipais de Meio Ambiente do Médio Piracicaba (MG). Ambiente E-Sociedade, $I 7(3), 239-258$.

Gabaldón, A. J. (2009). La descentralización de la gestión ambiental en América Latina. Reforma y Democracia, 45, I-I7.

Gibson, C. C. y Lehoucq, F. (2003). The local politics of decentralized environmental policy in Guatemala. Journal of Environment and Development, I2(I), 28-49.

Guitart, D., Pickering, C. y Byrne, J. (2012). Past results and future directions in urban community gardens research. Urban Forestry and Urban Greening, II(4), 364-373.

Hirsch, P., N'Guyen, A., Adrian-Kalchhauser, I. y Burkhardt-Holm, P. (20I6). What do we really know about the impacts of one of the 100 worst invaders in Europe? A reality check. Ambio, 45(3), 267-279.

Kauneckis, D. y Andersson, K. (2009). Making decentralization work: A crossnational examination of local governments and natural resource governance in Latin America. Studies in Comparative International Development, 44(I), 23-46.

Larsen, P. B. (20II). Municipal environmental governance in the Peruvian Amazon: A case study in local matters of (in)significance. Management of Environmental Quality: An International Journal, 22(3), 374-385.

Larson, A. M. (2002). Natural Resources and Decentralization in Nicaragua: Are Local Governments Up to the Job? World Development, 30(I), I7-3I. 
Larson, A. M. (2003a). Decentralisation and forest management in Latin America: Towards a working model. Public Administration and Development, 23(3), 2II-226.

Larson, A. M. (2003b). Gestión forestal municipal de Nicaragua: ¿Descentralización de cargas, centralización de beneficios? En L. Ferroukhi (Ed.), La Gestion Forestal Municipal en America Latina. Costa Rica: Cifor/IDRC.

Larson, A. M. (2004). Formal decentralisation and the imperative of decentralisation 'from below': a case study of natural resource management in Nicaragua. The European Journal of Development Research, I6(I), 55-70.

Larson, A. y Ferroukhi, L. (2003). Conclusiones. En L. Ferroukhi (Ed.), Gestión Forestal Municipal en América. Bogor, Indonesia: Cifor/IDRC.

Larson, A. M. y Lewis-Mendoza, J. (2012). Decentralisation and devolution in Nicaragua's North Atlantic autonomous region: Natural resources and indigenous peoples' rights. International Journal of the Commons, 6(2), I79-199.

Larson, A. M. y Ribot, J. C. (2004). Democratic decentralisation through a natural resource lens: an introduction. The European Journal of Development Research, I6(I), I-25.

Larson, A. M. y Soto, F. (2008). Decentralization of Natural Resource Governance Regimes. Annual review of environment and resources, 33, 213-239.

Larson, A. M., Pacheco, P., Toni, F. y Vallejo, M. (2007a). The effects of forestry decentralization on access to livelihood assets. Journal of Environment and Development, I6(3), 25I-268.

Larson, A. M., Pacheco, P., Toni, F. y Vallejo, M. (2007b). Trends in Latin American forestry decentralisations: Legal frameworks, municipal governments and forest dependent groups. International Forestry Review, 9(3), 734-747.

Lemos, M. C. y Agrawal, A. (2006). Environmental governance. Annual review of environment and resources, 31, 297-325.

Lemos, M. C. y Farias De Oliveira, J. L. (2004). Can water reform survive politics? Institutional change and river basin management in Ceará, Northeast Brazil. World Development, 32(I2), 212I-2137.

Nascimento, D. T. y Bursztyn, M. A. (20II). Descentralização da gestão ambiental: análise do processo de criação de organizações municipais de meio ambiente no sul catarinense. Revista do Serviço Público, 62(2), I85-208.

Neves, E. M. (2012). Política ambiental, municípios e cooperação intergovernamental no Brasil. Estudos Avançados, 26(74), 137-150.

Pacheco, P. (2004). What lies behind decentralisation? Forest, powers and actors in Lowland Bolivia. European Journal of Development Research, I6(I), 90-I09. 
Paulson Priebe, M. E., Evans, T., Andersson, K. y Castellanos, E. (2014).

Decentralization, forest management, and forest conditions in Guatemala. Journal of Land Use Science, Io(4), 425-44I.

Perreault, T. (2005). State restructuring and the scale politics of rural water governance in Bolivia. Environment and Planning A, 37(2), 263-284.

Pickering, C. y Byrne, J. (2014). The benefits of publishing systematic quantitative literature reviews for $\mathrm{PhD}$ candidates and other early-career researchers. Higher Education Research and Development, 33(3), 534-548.

Poats, S. V. y Suárez, D. (2007). Descentralización y gobernanza ambiental en áreas protegidas de Carchi, Ecuador: Lecciones de la Reserva Ecológica El Ángel y el Bosque Protector Golondrinas. Revista Virtual REDESMA, I, 87-98.

Ravikumar, A., Andersson, K. y Larson, A. M. (2013). Decentralization and forestrelated conflicts in Latin America. Forest Policy and Economics, 33, 80-86.

Ribot, J. C. (2004). Waiting for democracy. The Politics of Choice in Natural Resource Decentralization. M. Schultz y E. Yaghmour (Eds.). Washington D.C.: World Resources Institute.

Ribot, J. C., Agrawal, A. y Larson, A. M. (2006). Recentralizing While Decentralizing: How National Governments Reappropriate Forest Resources. World Development, 34(II), I864-I886.

Rodríguez-Becerra, M. y Espinoza, G. (2002). Gestión ambiental en América Latina y el Caribe. Evolución, tendencias y principales prácticas. D. Wilk (Ed.). Washington D.C: Banco Interamericano de Desarrollo.

Rupprecht, C. D. y Byrne, J. A. (20I4). Informal urban greenspace: A typology and trilingual systematic review of its role for urban residents and trends in the literature. Urban Forestry and Urban Greening, I3(4), 597-6II.

Thompson, D. (2016). Community adaptations to environmental challenges under decentralized governance in southwestern Uruguay. Journal of Rural Studies, 43, 7I-82. Ticona, R. P., Vázquez, M. R., Cal y Mayor, A. B., y Meza, A. L. (2005).

Participación social y toma de decisiones en los consejos municipales de desarrollo rural sustentable de Los Altos de Chiapas. Gestion y Politica Publica, I4(2), 34I-402.

Uberhuaga, P., Larsen, H. O. y Treue, T. (20II). Indigenous forest management in Bolivia: Potentials for livelihood improvement. International Forestry Review, I3(I), 80-95.

Wilder, M. y Romero Lankao, P. (2006). Paradoxes of Decentralization: Water Reform and Social Implications in Mexico. World Development, 34(II), I977-I995.

Wittman, H. y Geisler, C. (2005). Negotiating locality: Decentralization and communal forest management in the Guatemalan highlands. Human Organization, $64(\mathrm{I}), 62-74$. 


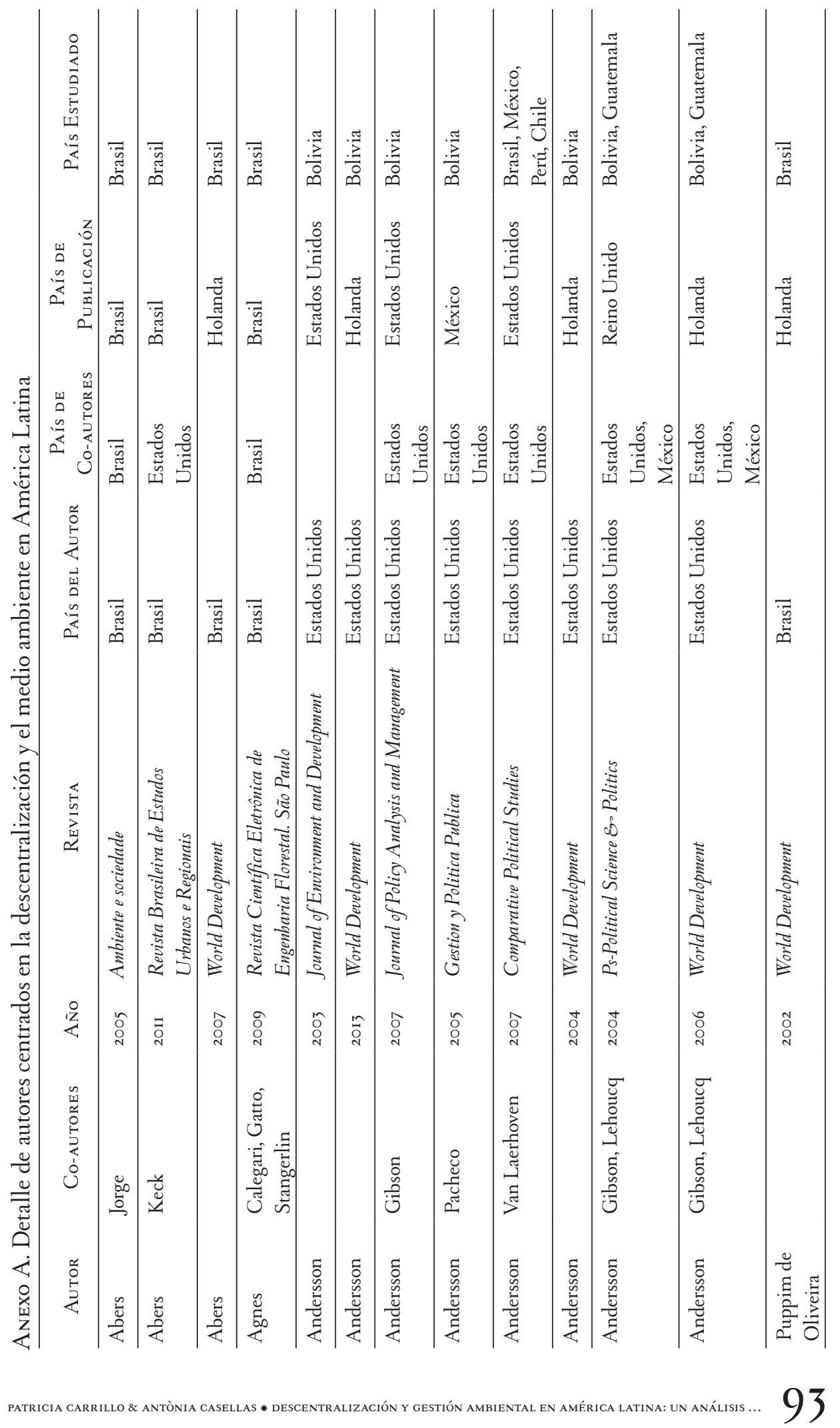




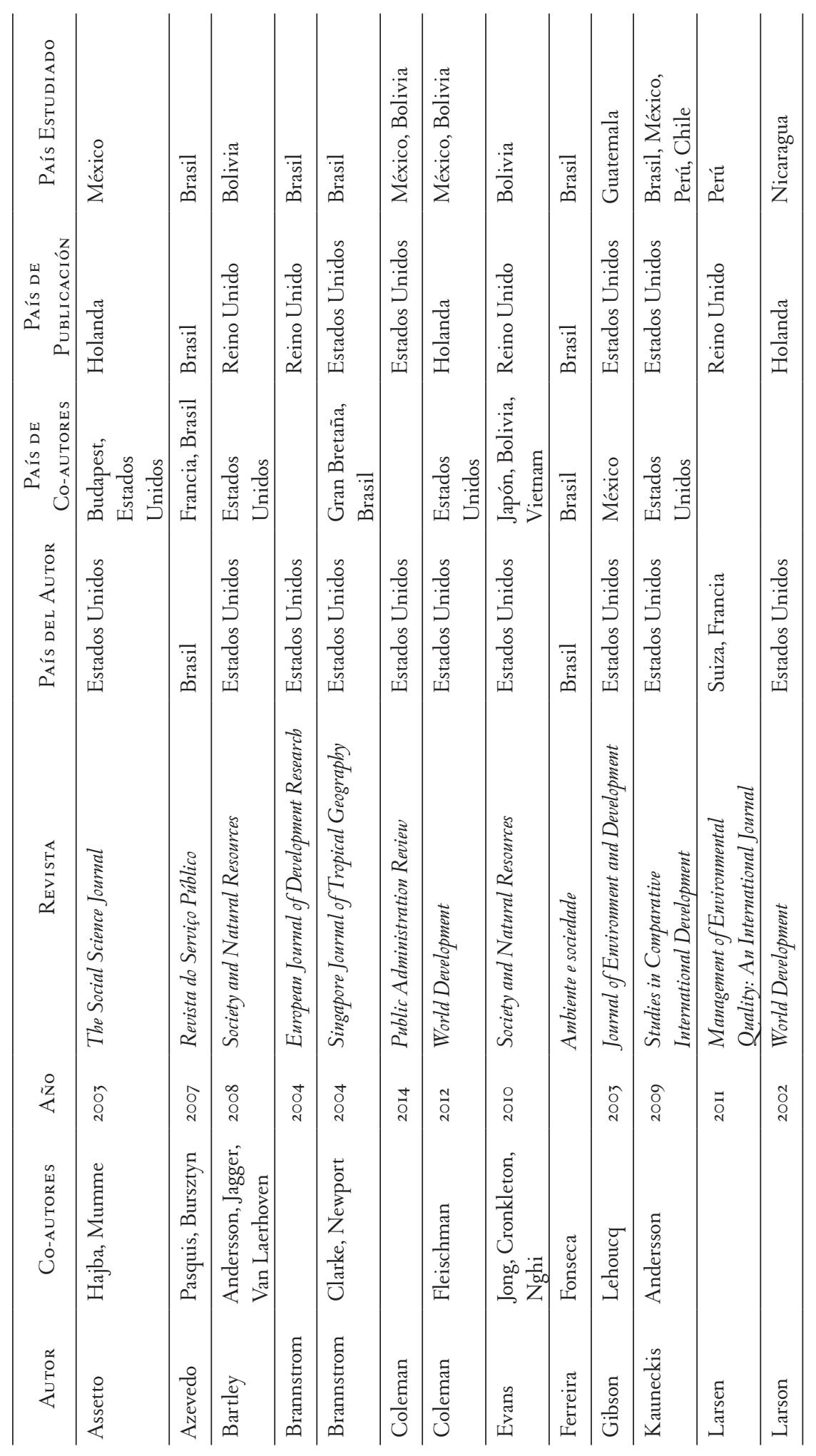




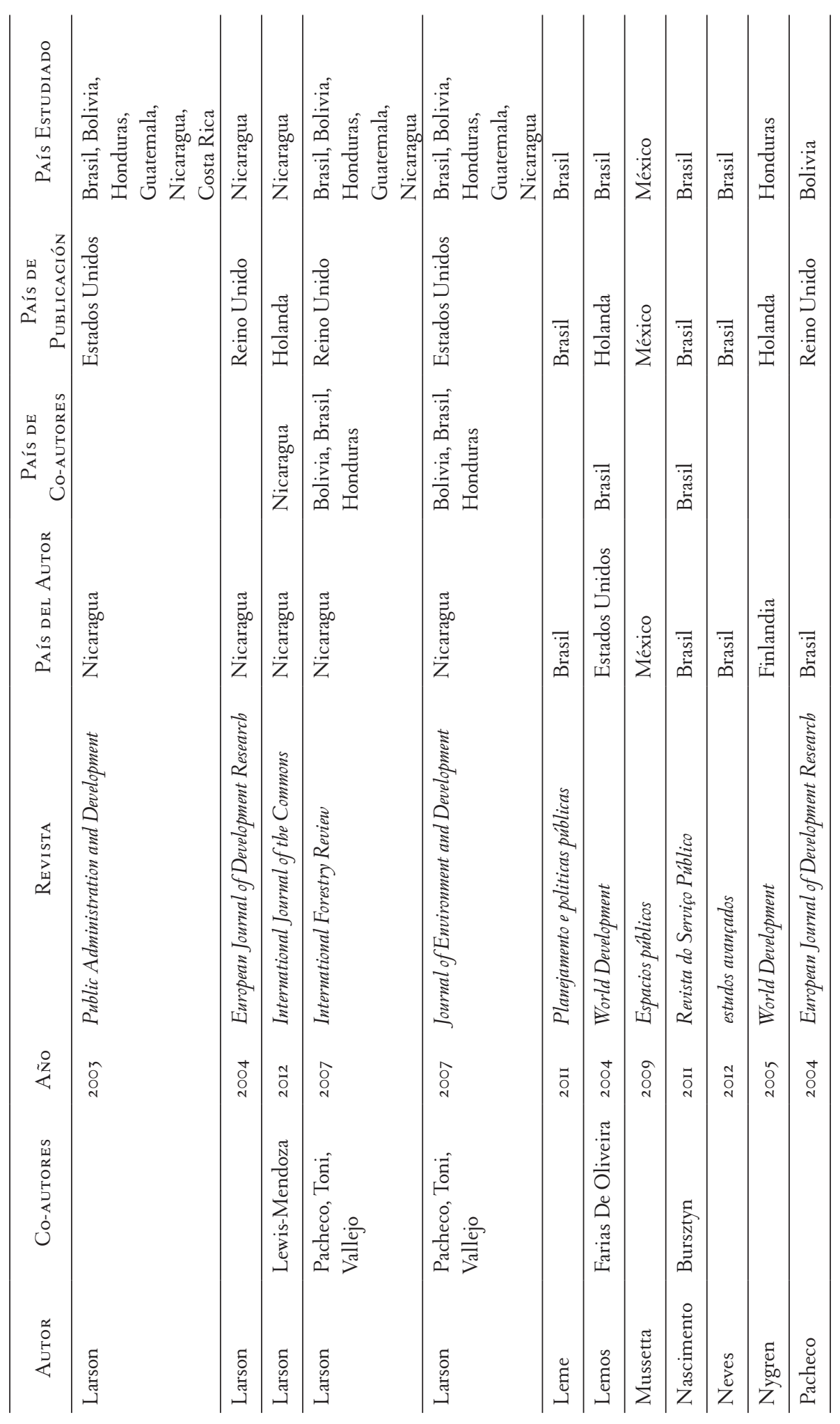

PATRICIA CARRILLO \& ANTÒNIA CASELlAS * DESCENTRALIZACIÓN Y GESTIÓN AMBIENTAL EN AMÉRICA LATINA: UN ANÁLISIS ... 


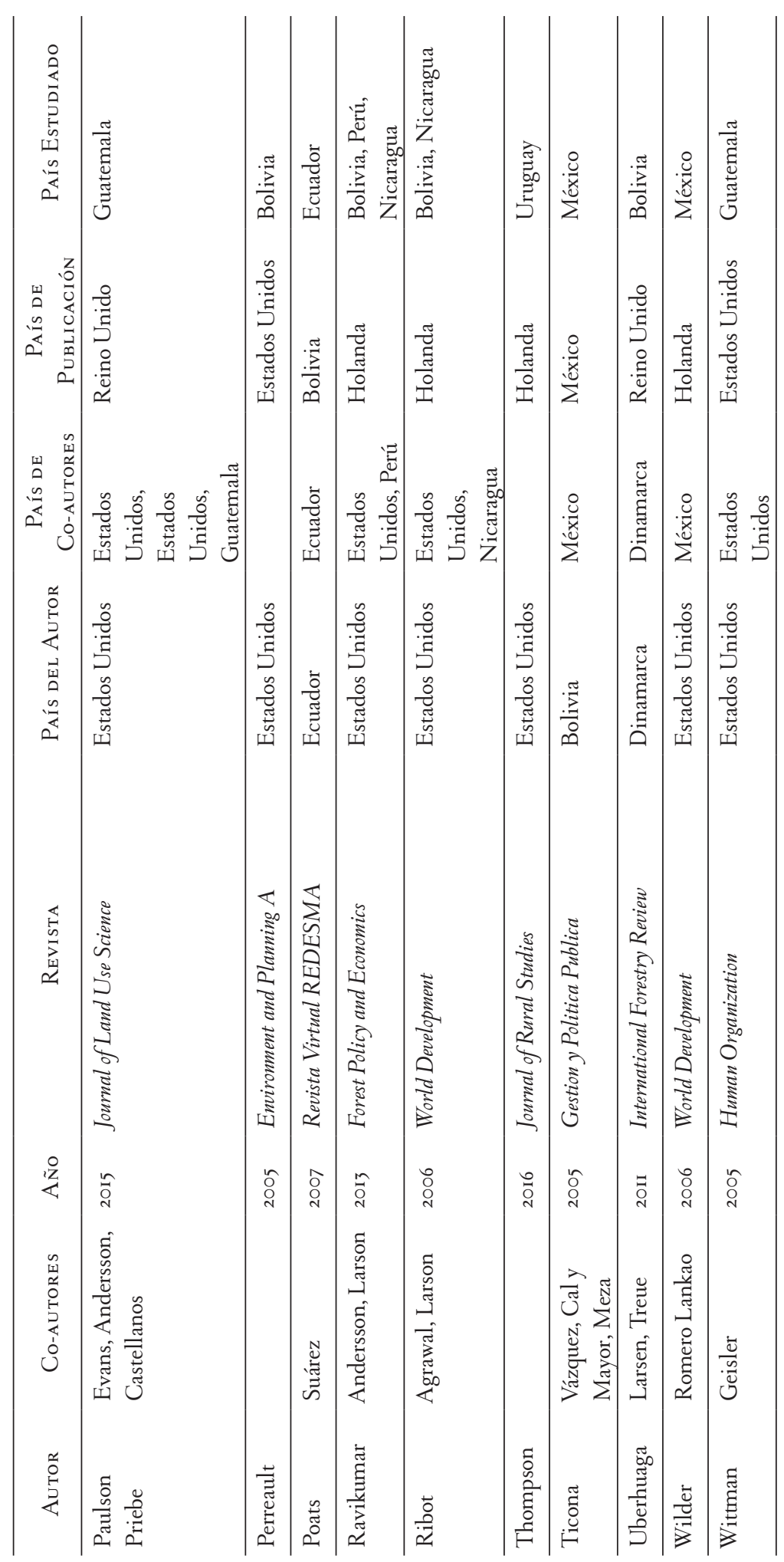

